\title{
NEW STRUCTURED PACKING CUB FOR PURIFICATION OF EXHAUST GASES
}

\author{
Irina NOVIKOVA, Alexander PUSHNOV ${ }^{2}$ \\ Moscow State University of Mechanical Engineering, Moscow, Russia \\ E-mails: ${ }^{1}$ irrrinochka93@mail.ru; ${ }^{2}$ pushnovas@gmail.ru
}

\begin{abstract}
New structured packing for heat and mass transfer processes named CUB is presented in our article. The packing can be applied in packed towers for exhaust gas cleaning instead random packing, for example, rings type that are the most used in such processes. The advantages of the new packing over random packing are lower pressure drop, capability of purification and as a consequence long-term service of the packing. The researches of intensity of liquid-phase mass-transfer in packed bed depending on liquid spray rate and gas velocity were carried out. Obtained data show that packing CUB is more effective than the most popular type of structured packing under all other conditions being equal. As experimental data shown heat transfer coefficient was up by $17 \%$ and mass transfer coefficient was up by $51 \%$. We found out optimal geometry of cross section of the new packing, namely, number of elements and parameters of one element. The new construction of structured packing is applicable for both type of column cross-section round and square.
\end{abstract}

Keywords: structured packing, waste gas purification.

\section{Introduction}

Object and relevance of research. The polluting emissions protecting of wildlife resources and airspace is connected with the range of ecological problems. The solution of these problems ensures the whole planet safety. The intensive development of industrial production promotes amplification and variety of technologies that are connected with emission into the atmosphere of pollutants. Food and light industry enterprises, integrated iron-and-steel works, petroleum processing plants, cement and chemical productions - all of them necessitate gas- and dust separation equipment. Increased productive capacity dictates the new ideas development of gas purification plants perfection (Bolshakov et al. 2000). Scrubbers of various designs are widely applied for cooling of process gas at enterprises of chemical, petrochemical, cryogenic, metal and other industries. The process purpose can be different:

1) liquid absorption some sort of gas component,

2) gas purification from dust or particulate,

3) gas humifying,

4) cooling or heating of gas (contact heat transfer).

The scrubber is filled packing through that liquids flow in the form of films. The feed gas moves as counter

flow. Wetted packing surface is the contact surface of interacting phases. There are two types of packing: random and structured (Berengarten et al. 2014). Both have advantages and disadvantages. The object of our research is the new structured packing, which is more effective than the most used type of structured packing.

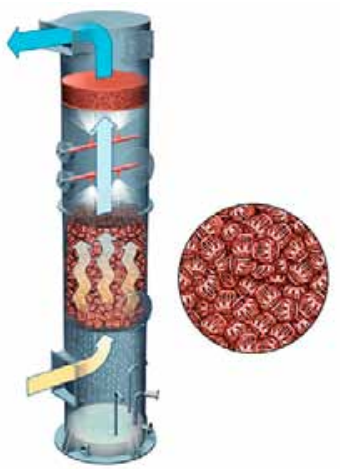

Fig. 1. The scheme of scrubber with random packing

\section{Literature review}

Researches of intensification heat and mass transfer in structured packing columns have being carried out in our university for several years now.

In doctoral dissertation (Dmitrieva 2007) vertical separation of adjacent corrugated packing blocks by inserts to create turbulation was proposed. This approach was developed in dissertation (Kharitonov 2013). Author 
presented creating turbulisation way in the form of vertically alternated blocks of corrugated sheets with lattice prisms layers.

Successful effort to eliminate the main disadvantages, namely lesser intensity of fluid film renewal, is undertaken in dissertation (Tsurikova 2013). Intensification of heat and mass transfer process was achieved by entered hydrodynamic effects use in this work. Structured packing block was made in form of particular short layers. These layers are interruptedly disposed height along and are turned 90 degrees relatively to each other in horizontal plan as it is shown in Figure 2. As test data shown, such packing construction increased mass transfer coefficient comparison with traditional structured packing. Optimal height of SLP was found out.

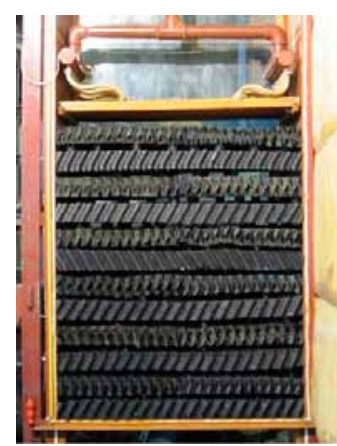

Fig. 2. Structured packing block made in form of particular short layers

\section{The review of two packing types}

Clean packing of dust is very time-consuming process, which is connected with tacking-out the packing from apparatus. That's why using structured packing instead random packing is more rationality for purification gases.

So, as it can be seen in Figure 3, structured packing have less pressure drop in comparison with random packing such as Raschig rings (Fig. 4), Pall rings (Fig. 5), Bialecki rings (Fig. 6). Pressure drop reduction decreases energy demands of purification equipment operation.

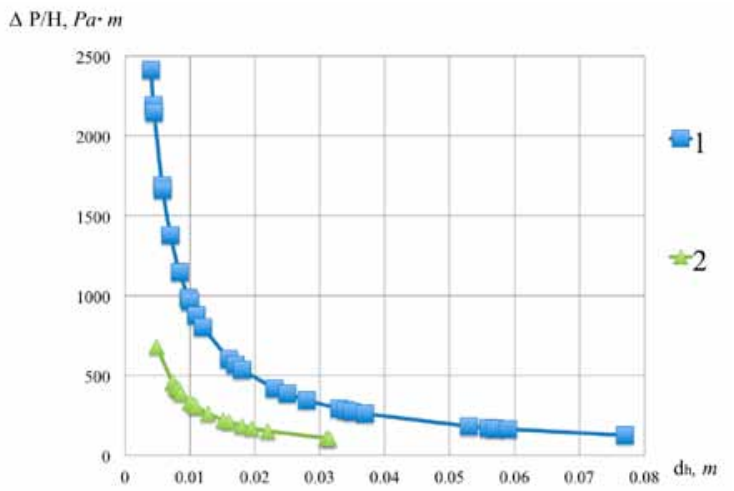

Fig. 3. Pressure drop as a function of hydraulic diameter for different types of 1 - random packing; 2 - structured packing

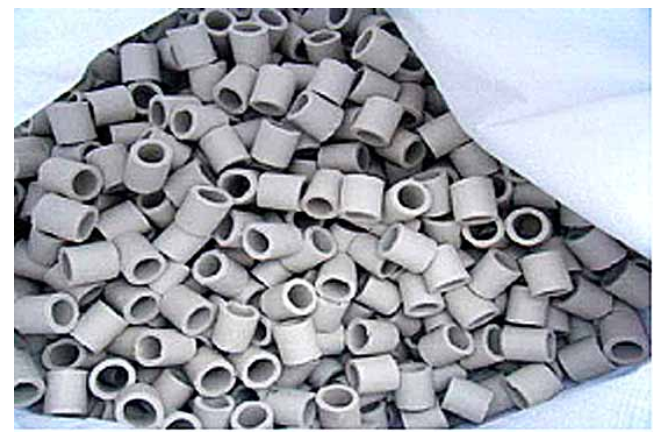

Fig. 4. Ceramic Raschig rings

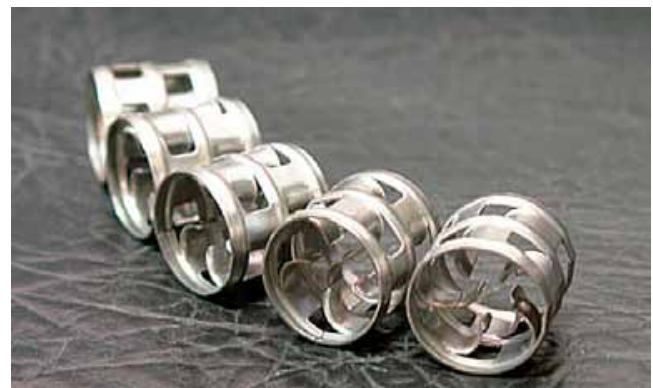

Fig. 5. Metal Pall rings

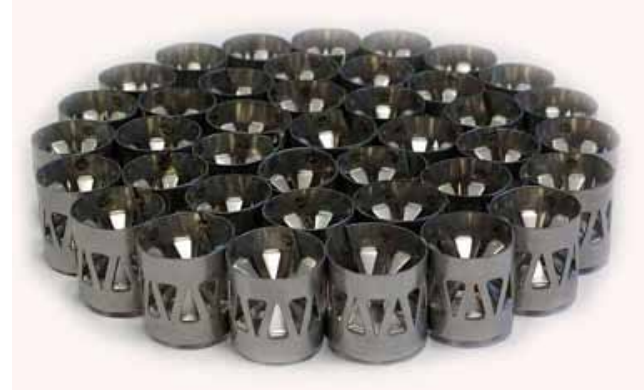

Fig. 6. Metal Bialecki rings

However, albeit such important advantages, structured packing has two fundamental disadvantages in comparison with random packing:

1) lesser frequent disruption of liquid film, which is an important factor for intensification heat and mass transfer processes;

2) side-to-side mixing absence between adjacent corrugated sheets in the packing block volume (Pushnov, Kagan 2011).

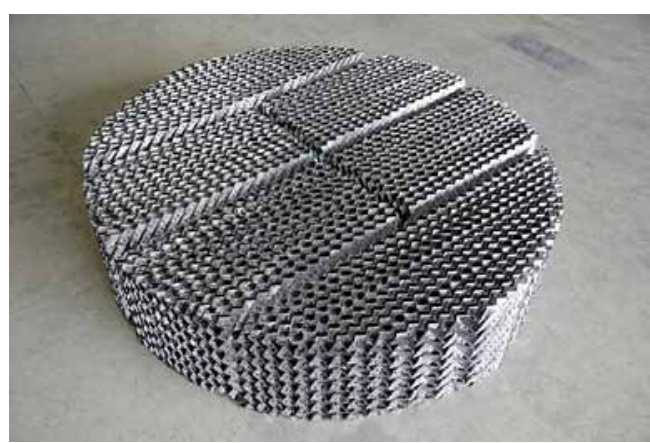

Fig. 7. The example of metal structured packing 


\section{Ways to decrease structured packing disadvantages and the new packing conception}

The further improvement of structured packing that is applied in purification processes is directly connected with decreasing of two noticed disadvantages. The first disadvantage, namely, less frequent disruption of liquid film, is solved by packing make in the form of short layers.

The second disadvanatage of structured packing is decreased by packing cross-section geometry change. Instead traditional structured packing form, proposed packing is several short layers (Fig. 8) and each of these layers is some cubical elements (Fig. 9). Dimensions of each cubical elements is dictated by column apparatus diameter. Cubical elements fully fill apparatus cross-section. Fields near column walls is filled by segmental elements in the case of round column. Cubical elements are turned by 90 degrees relativey to each other in horisontal plan. Each next short layer is rotationally layed relatively to the previouse layer.

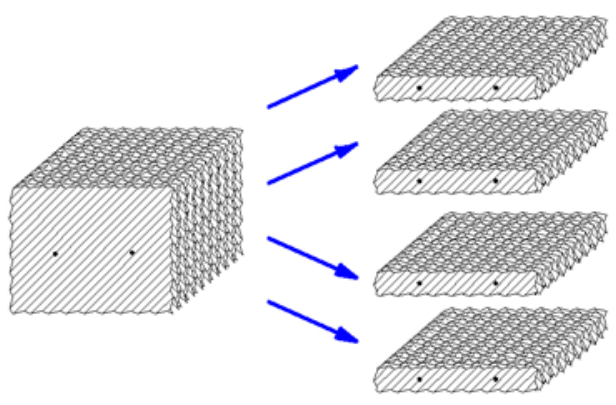

Fig. 8. Packing block in the form of short layers

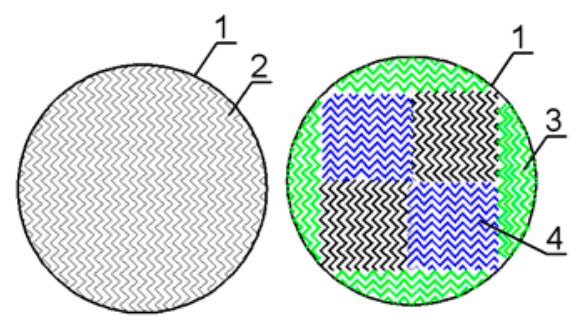

Fig. 9. Cross-section geometry of the packing block changing.

(1) - column apparatus, (2) - standard regular packing,

(3) - segmented elements, (4) - cubical elements

Evidently, the minimum amount of cubical elements is 4 . But the more cubical elements fill the column cross-section the more side-to side mixing increases. This is explained by expansion in the number of zones, wherein vortex-flow formation occurrences.

In Figure 11 comparison schemes of gas-flow behavior moving in two packing types, namely in the standard structured packing and in the proposed packing CUB.

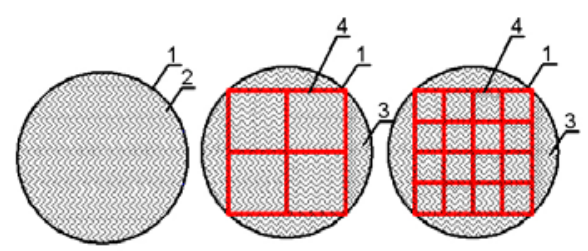

Fig. 10. Cross-section geometry of the packing block changing. (1) - column apparatus, (2) - standard regular packing, (3) - proposed packing CUB, (4) - zones of vortexflow formation

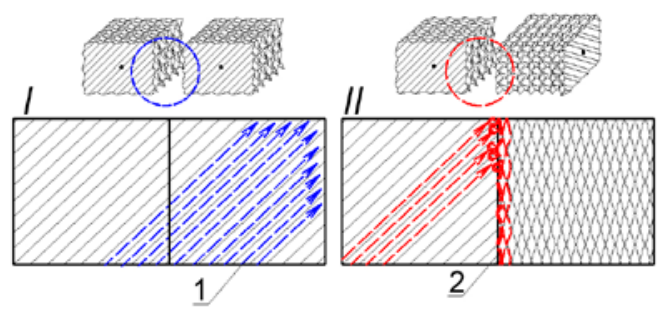

Fig. 11. Gas flow behavior at the junction of adjacent cubical elements (I) - gas flow in the regular structured packing,

(II) - gas flow in packing CUB, (1) - gas flow motion trajectory, (2) - vortex activity (turbulence)

As we can see in this picture there is just streamline gas motion in the channels between corrugations in the case of standard structure packing (the left part of Fig. 10). In the case of CUB at the beginning there is streamline gas motion in the channels too. But then, the gas stream meets with the hindrance in the form of orthogonally layed sheets of the block number two slows down and turns up across corrugations flowing around the hindrance.

At that: flow turbulisation occurs and residence time of interacting gas-fluid streams in the packing volume increases. As a result, of heat and mass transfer processes can be expected.

\section{Methodology}

At the first step of our research we compared the prototypes of the standard packing block type of Mellapak and proposed packing CUB. Hydraulical tests were carried out (Novikova, Pushnov 2015).

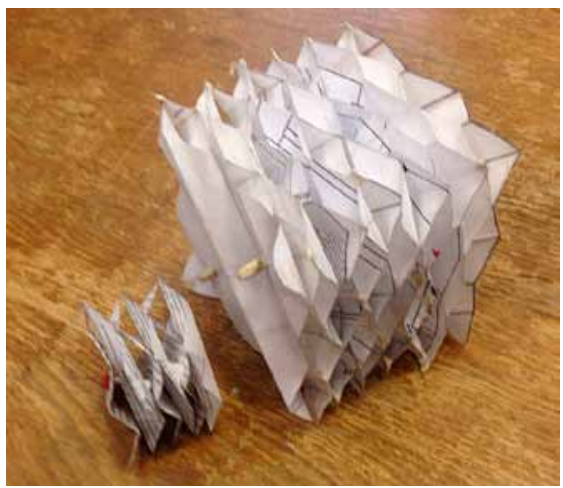

Fig. 12. Paper models of standard block and element of CUB 
Firstly we tested paper models. Obtained data shown that dry pressure drop of CUB was more than pressure drop of standard block. Then we tested metal models of standard, short layered packings and CUB. Pressure drop of CUB was the biggest again. Pressure drop of SLP was lesser than CUB and standard block had the lowest pressure prop.

In this work we presented the second step of our research, namely research of heat and mass transfer processes in CUB.

Effectiveness of proposed packing construction was checked in the laboratory stand, which is presented in Figure 13.

Two types of packing were compared:

1) short layered packing (Fig. 14);

2) proposed packing CUB (Fig. 15).

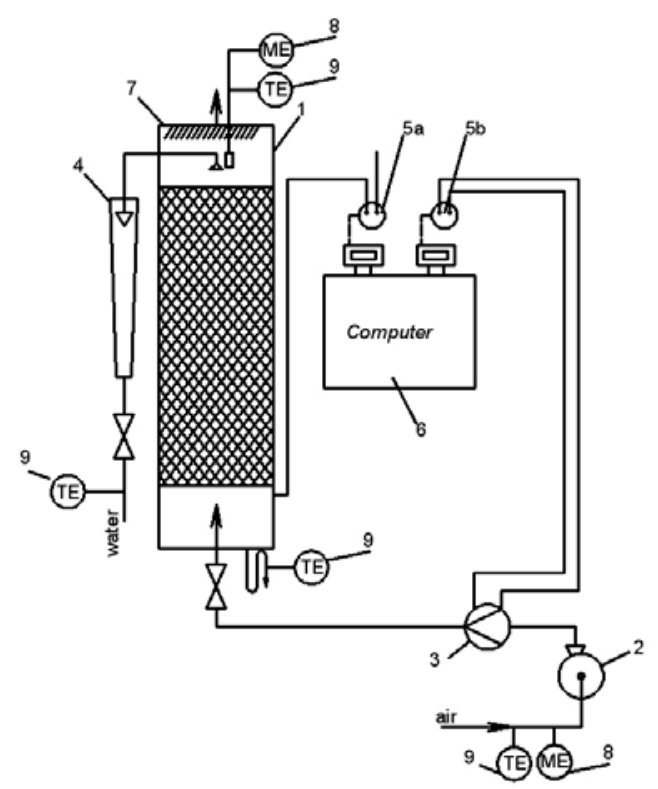

Fig. 13. The experimental stand. (1) - packed column, (2) - fan, (3) - airflow measuring device, (4) - liquid flowmeter (rotameter), (5) - faucets a and b, differential manometers (5a) and (5b), (6) - computer, (7) - spray separator, (8) - humidity meters, (9) - thermal couples

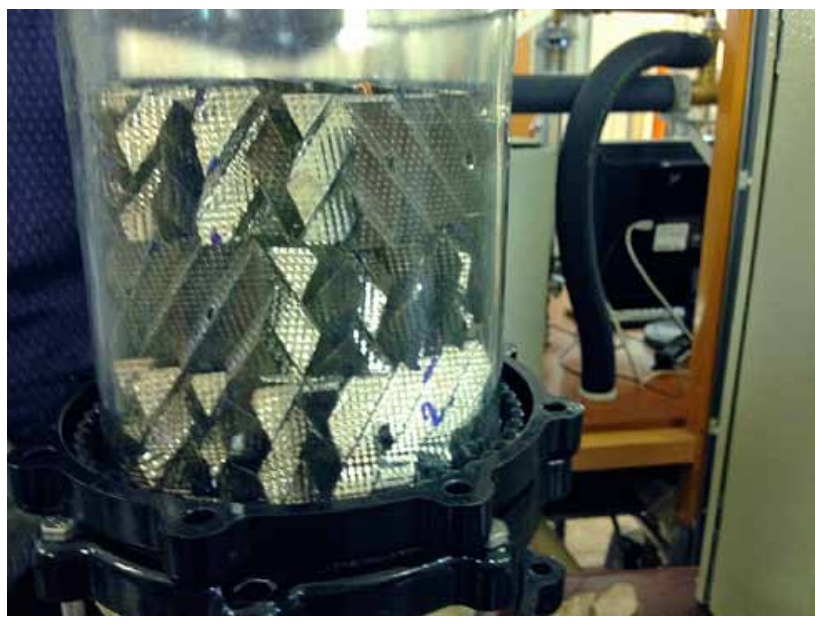

Fig. 14. Short layered packing in the column apparatus

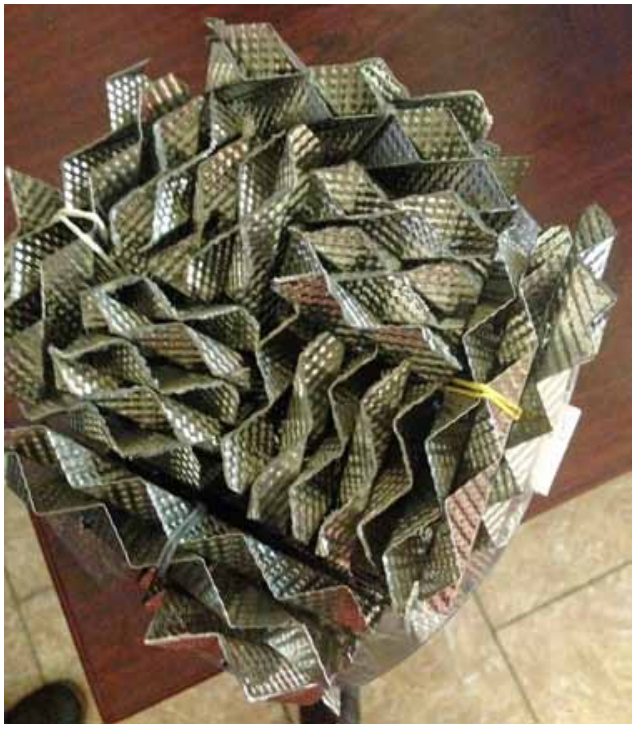

Fig. 15. Proposed packing CUB

Geometrical characteristics of two packing types are listed in Table 1.

Table 1. Geometrical characteristics of tested packing prototypes

\begin{tabular}{|l|c|c|}
\hline & CUB & $\begin{array}{c}\text { Short layered } \\
\text { packing }\end{array}$ \\
\hline Specific surface, $\mathrm{m}^{2} / \mathrm{m}^{3}$ & 159.1 & 169.4 \\
\hline Void fraction, $\%$ & 76 & 69.9 \\
\hline Hydraulic diameter, $\mathrm{m}$ & 0.0192 & 0.016 \\
\hline Packing bed height, $\mathrm{m}$ & .23 & 0.24 \\
\hline
\end{tabular}

Testing of the packings was carried out in the water-air system. Spray packings were tested and water heating process under direct interaction with air in counter-current flow was carried out. Liquid spray rate was $310 \mathrm{~m}^{3} /\left(\mathrm{m}^{2} \cdot \mathrm{s}\right)$ in hydraulic and heat and mass transfer testings. Superficial gas velocity was from $0.21 \mathrm{~m} / \mathrm{s}$ to $1.19 \mathrm{~m} / \mathrm{s}$. Airflow rate, water rate, pressure drop of dry and spray packing beds were detected when hydraulic testing. Airflow rate, water rate, temperatures at input of the air and water, temperatures at output of the air and water, humidity of the air at input and output were detected when heat and mass transfer testing.

\section{Obtained results}

Dependents sprayed specific pressure drop $\Delta \mathrm{P} / \mathrm{H}$ of superficial gas velocity $\mathrm{w}_{0}$ for both types of tested packing are presented in Figure 16. Testing of based packing (SLP) and proposed packing (CUB) were carried out in the laboratory stand under the same conditions.

As we can see in Figure 16, specific spryed pressure drop of packing CUB is rather more than short layered packing (SLP) within number of gas velocity from 0.8 to $1.2 \mathrm{~m} / \mathrm{s}$. 


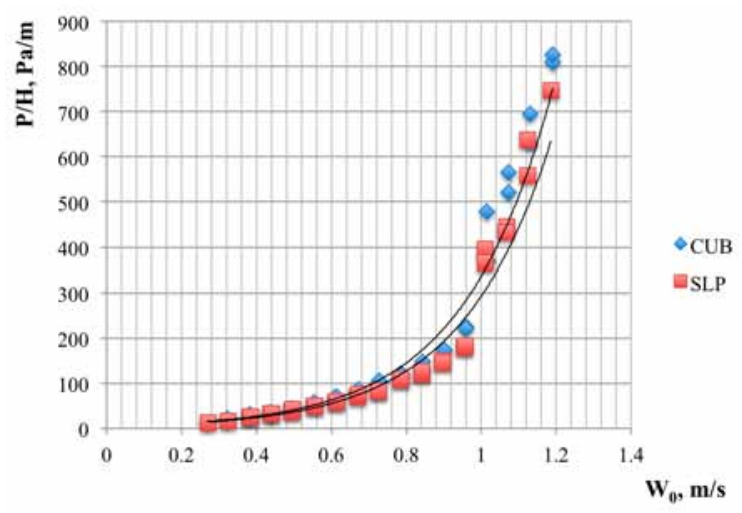

Fig. 16. Experimental dependents spayed specific pressure drop of superficial gas velocity for both types of tested packing

When gas velocity is $1.18 \mathrm{~m} / \mathrm{s}$ pressure drop of CUB is higher by $13.8 \%$ than SLP pressure drop.

Dependents heat transfer coefficient of superficial gas velocity for both types of tested packings is shown in Figure 17. This suggests that the proposed construction allows increasing mass heating transfer coefficients. Heat transfer coeffiicent of CUB is higher by $17.24 \%$ than heat transfer coefficient of SLP when superficial gas velocity is $1.18 \mathrm{~m} / \mathrm{s}$.

Dependents mass transfer coefficient of superficial gas velocity for both types of tested packings is shown in Figure 18.

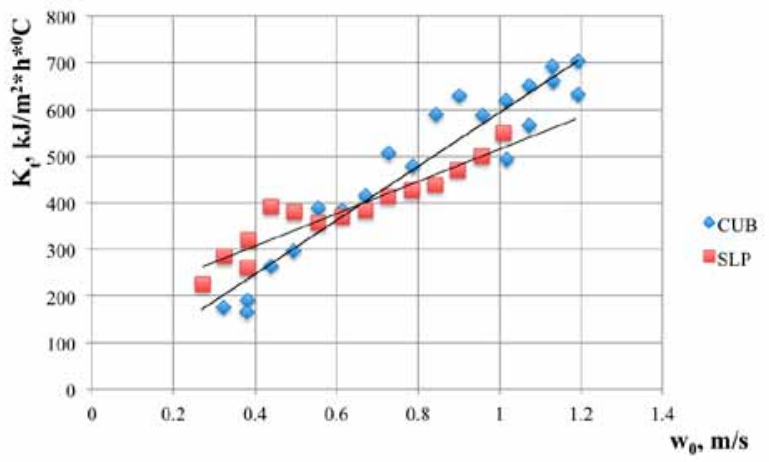

Fig. 17. Experimental dependents heat transfer coefficient of superficial gas velocity for both types of tested packing

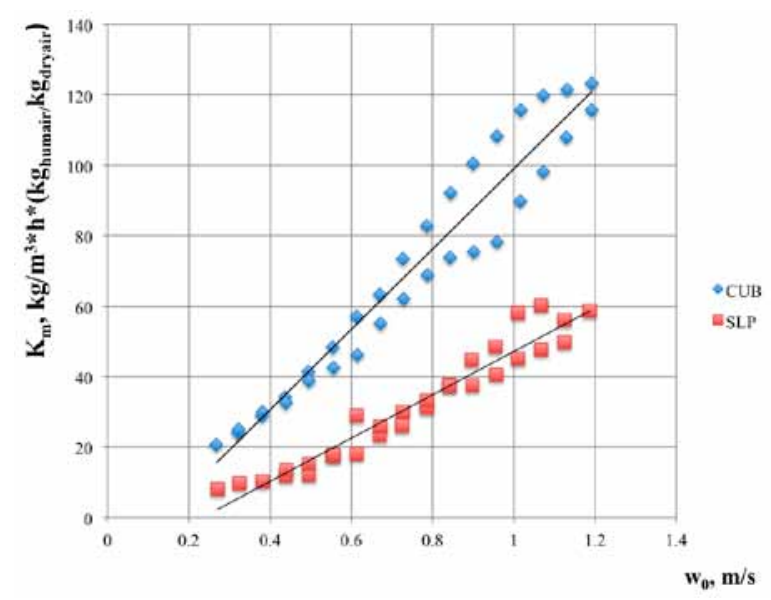

Fig. 18. Experimental dependents heat transfer coefficient of superficial gas velocity for both types of tested packing
Mass transfer coefficient of CUB is higher by $51.3 \%$ than mass transfer coefficient of SLP when superficial gas velocity is $1.18 \mathrm{~m} / \mathrm{s}$.

So, we have the following pattern: turbulence increasing leads to pressure drop increasing, at it is shown in Figure 16. In turn the turbulence increasing intensifies heat and mass transfer process. The ability of correlation of pressure drop $\Delta \mathrm{P}$ (and therefore turbulence number $\mathrm{Tu}$ ) and the growth of certain heat and mass transfer process effectiveness is the following task of our researches.

\section{Conclusions}

1. The use of proposed structured packing allows increasing side-to-side mixing gas phase between adjacent corrugated sheets in the block packing volume by additory flow turbulence. Proposed laying adjacent elements of packing CUB that are turned by 90 degrees relatively to each other in horizontal plan creates side to side mixing gas phase by vortex-like flow. It admits to eliminate the mentioned disadvantage of structured packing.

2. Comparative tests of proposed and based packings were carried out. The investigations proved the increasing of heat and mass transfer effectiveness due to proposed construction of the new packing CUB.

3. It is showed that using packing CUB allows to increase heat transfer coefficient by $17.24 \%$ and mass transfer coefficient by $51.29 \%$ under gas velocity $1.18 \mathrm{~m} / \mathrm{s}$.

4. Now packing CUB is in the patenting process.

\section{References}

Bolshakov, V. N.; Lipunov, I. N.; Lobanov, B. I. 2000. Ecology: book for technical colleges. M.: Intermet Ingineering. 330 p. ISBN 5-89594-036-6.

Berengarten, M. G.; Vitkovskaya, R. F.; Gorodilov, A. A.; Pushnov, A. S. 2014. Processes and apparatuses of protecting enviroment. Packing scrubbers for contact heat transfer. Saint Petersburg. 82 p.

Dmitrieva, G. B. 2007. Hydrodynamics and mass transfer in structured packing of corrugated sheets: Doctoral dissertation. Ivanovo State University of Chemistry and Technology, Ivanovo. 255p.

Kharitonov, A. A. 2013. Evaporation cooling in structured combination contact devices of cooling tower: Doctoral dissertation. Ivanovo State University of Chemistry and Technology, Ivanovo. $160 \mathrm{p}$.

Novikova, I. V.; Pushnov, A. S. 2015. Concept of the new structured packing for heat and mass transfer processes. Advantages in heat transfer, in Proceeding of the $7^{\text {th }}$ Baltic Heat Transfer Conference, 24-26 August 2015, Tallin, Estonia. 213 p.

Pushnov, A. S. Kagan, A. M. 2011. The structured and hydrodynamic of packing column apparatuses. Saint Petersburg. $135 \mathrm{p}$.

Tsurikova, N. P. 2013. Influence the high of block on structured packing to evaporation cooling in mechanical-draft cooling tower: Doctoral dissertation. Moscow. 131 p. 